\title{
Experimental investigation of milling regimes in planetary ball mill and their influence on structure and reactivity of gasless powder exothermic mixtures
}

\author{
A.S. Rogachev ${ }^{1,2^{*}}$, D.O. Moskovskikh ${ }^{1}$, A.A. Nepapushev ${ }^{1}$, T.A. Sviridova ${ }^{1}$, S.G. Vadchenko ${ }^{2}$, \\ S.A. Rogachev ${ }^{2}$, A.S. Mukasyan ${ }^{3}$ \\ ${ }^{1}$ National University of Science and Technology, "MISIS", Moscow, 119049, Russia \\ ${ }^{2}$ Institute of Structural Macrokinetics and Materials Science, RAS, Chernogolovka, 142432 Russia \\ ${ }^{3}$ Department of Chemical and Biomolecular Engineering, University of Notre Dame, Notre Dame, IN, \\ 46556 USA \\ *Corresponding author, e-mail: rogachev@ism.ac.ru
}

\begin{abstract}
.
The dynamic of ball motion during high energy planetary ball milling was investigated in-situ using high speed video recording. The trajectories and velocities of the balls were determined for different milling conditions. Correlations of these parameters, with both microstructure and reactivity of various as-milled powder mixtures ( $\mathrm{Ni}-\mathrm{Al}, \mathrm{Ti}-\mathrm{Si}, \mathrm{Si}-\mathrm{C})$, were revealed. It was shown that all of the main theoretically predicted milling regimes - cascading, cataracting and centrifugal - can be achieved by varying the rotation speed of the vial. The conclusion is made that shear deformation plays a major role in the transformation of the microstructure and reactivity of these powder mixtures. According to this observation, the cascading regime appears to be the most effective for controlling the properties of the mixtures.
\end{abstract}

Key words: high energy ball milling, mechanical activation, planetary ball mill, ball motion, high energy density materials, SHS

\section{Introduction}

High-energy ball mill (HEBM) is widely used for grinding, alloying and mechano-chemical synthesis of various materials, including nanomaterials [1-3]. It was also found that HEBM of reactive systems (i.e. mixtures of two or more powders that can react in a self-sustained mode) results in a decrease of the reaction onset temperature and an increase of the reaction rate [4-7]. Thus, it is concluded that such a mechanical treatment "activates" the reactive systems. During the last 15 years, special attention was paid to, the so-called, mechanically activated self-propagating high-temperature synthesis (MA-SHS) [8-12]. SHS reactions allow for the fabrication of different materials by means of the "solid flame" (gasless) type of processes in a heterogeneous mixture of powder reactants (cf., [13, 14]). An essential requirement for such reactions is that they must generate enough heat in order to proceed in the self-sustained mode. The combination of HEBM and SHS significantly expands the 
capabilities of both methods, because such a mechanical treatment decreases the critical threshold for heat release. Weakly exothermic mixtures (e.g., $\mathrm{Si}+\mathrm{C}$ ), for which the self-sustained reaction could not be accomplished under conventional conditions, become combustible after HEBM [15]. The mechanism of this effect is still not well understood. Our recent investigation suggests that structural modification of the reactive media, including both nano-scale mixing of the reactants and sometimes the formation of nuclei of new phases during HEBM, may be responsible for the enhancement of reactivity in the $\mathrm{Ni}+\mathrm{Al}$ and $\mathrm{Ti}+\mathrm{C}$ systems $[16,17]$. Severe deformation of metals and the formation of sub-micrometer, or nano-scale, composite particles during ball milling have been observed in many works $[9,10,12]$. In order to understand how these HEBM-induced structures formed, it is critical to know the parameters of the ball movement in the grinding vessel.

The trajectories of the balls inside the vessel during HEBM were studied in numerous theoretical and few experimental works. According to theoretical analyses, depending on the rotation speed, friction coefficient, ball diameter, and other parameters: the balls can roll and slide along the inner surface of the grinding chamber, move along free-fall trajectories, make periodic circular, or chaotic swirling motions (cf. [18-20]). A variety of parametric maps were modeled to show the correlations between the trajectories of the balls and planetary mill rotation regimes (cf. [21]). Unfortunately, the validation of these theories is hardly possible due to the lack of direct experimental data on the real ball behavior during milling. Moreover, the available experimental observations often contradict the theoretical predictions. For example, most of the models consider impacts between the balls as the main factor, which influences the powder structure. However, the first direct photo- and high speed video-recording of the balls motion led to the conclusion that the HEBM process should be primarily described in terms of attrition and wear and not in terms of impact [22]. For example, the direct high-speed video recording of the ball motion made by the camera installed on the rotating sun wheel above the jar, showed only rolling and sliding of the balls for all investigated parameters of the considered planetary mill, while the free falling regime has not being observed [23]. 
In this work we study the ball motion in-situ in the planetary mill, depending on the parameter $K=w / W$, where $w$ and $W$ are the rotation speeds of the grinding vessel and the sun wheel, respectively. High speed video recording, followed by frame by frame computer treatment of the obtained movies, allows us to retrieve the trajectories of the balls and measure both their instantaneous velocities and accelerations for different milling conditions. The same HEBM regimes were applied to three separate binary reactive powder mixtures, i.e. Ni-Al (ductile-ductile), Ti-Si (ductile-brittle) and Si-graphite (brittle-brittle). The morphology and reactivity of these systems were investigated as a function of parameter $K$. The correlations between the planetary ball mill regimes and the properties of the asmilled reactive powder mixtures were identified and discussed.

Thus, the goal of this work is to obtain direct experimental data on the correlations between the different high energy planetary ball milling regimes of the reactive powder mixtures, the morphology of as-milled particles, and the reactive properties of these mixtures.

\section{Experimental procedure}

A laboratory-scale planetary ball mill "Activator-2S" (Novosibirsk, Russia) was used in this work. Two vertical grinding steel vessels (vials), with inner volumes of $250 \mathrm{ml}$ apiece, are placed on the sun wheel, as shown at Figure 1. Two independent electric motors drive the sun wheel and the vials with the rotating rates $\mathrm{W}$ and $\mathrm{w}$, correspondingly. A computer controls the rotation speed through two frequency converters. The operational parameters used in this research are listed in Table 1. Note that for this mill, the rotating directions of the sun wheel and the grinding vials are opposite, therefore, the parameter $\mathrm{K}=\mathrm{w} / \mathrm{W}$ has a negative value. However, for the sake of convenience, we use an absolute value of the rotating velocities for determination of $\mathrm{K}$ value. As can be seen from Table 1 we kept the velocity of sun wheel constant $(\mathrm{W}=694 \mathrm{rpm})$ and changed $\mathrm{K}$ by varying the velocity of the vial (w) from 0 to $1388 \mathrm{rpm}$. All other parameters remained constant during all experiments. The values of the pre-set rotating speeds were verified with the high-speed video camera. 
In the video recording experiments, the steel lids of the vials were replaced with transparent acrylic glass lids, and four LED lamps were used for illumination of the sun wheel and the vials. The high speed video camera Phantom Miro M310 (Vision Research Inc., USA) was placed above the mill, and the lens of the camera was directed along the vertical axis of the sun wheel. The video frame at the Figure 1a represents the view field of the camera. A recording rate of 2000 frames per second was used in all experiments. In order to monitor the trajectories of the balls inside the grinding vessels, computer treatment of the video records was made. Two square windows were selected, as shown at Figure 1b. These windows rotated around the sun wheel axis with the same rate as the grinding vials. Thus, axis of each vial remained stationary relative to the window, i.e. as if the video camera was physically installed at the sun wheel on the top of the vial. This procedure was accomplished by use of a specially developed computer program, written in $\mathrm{C}++$ using the QPainter class rotate procedure from the Qt Library. Each window has dimensions of $358 \times 358$ pixels and an optical resolution of $0.273 \mathrm{~mm}$ per pixel. Examples of the selected images inside the windows are shown at Figure 2. The coordinates $(\mathrm{X}, \mathrm{Y})$ of the balls on each frame were measured by using an image processing program (Irfan View). Based on the data obtained the trajectories of the balls, their instantaneous velocities and accelerations were calculated.

Three reactive powder mixtures were chosen for the investigation of the HEBM regimes and their influence on their structures and reactivity: $\mathrm{Ni}+\mathrm{Al}, \mathrm{Ti}+0.6 \mathrm{Si}$ and $\mathrm{Si}+\mathrm{C}$. These SHS-systems represent three types of the powder mixtures: ductile $(\mathrm{Ni})+\operatorname{ductile}(\mathrm{Al})$, ductile $(\mathrm{Ti})+$ brittle (graphite) and brittle $(\mathrm{Si})+$ brittle (graphite). Initial powder mixtures were prepared using the following powders: Ni (PNK, purity $99.9 \%$, average particles size $\mathrm{d} \sim 20 \mu \mathrm{m}$ ), Al (ASD-1, $99.7 \%$, average $\mathrm{d} \sim 20 \mu \mathrm{m}$ ), Ti (PTM, 99\%, average $\mathrm{d} \sim 40 \mu \mathrm{m}$ ), Si (KEF-4.5, purity $>99,9 \%$, $\mathrm{d}<63 \mu \mathrm{m}$ Russian State Standard 19658) and C (graphite, Alfa Aesar, purity, 99\%, d < $10 \mu \mathrm{m}$ ). The powders were weighed in the desired amounts and were placed in the steel grinding vials together with the steel balls; the mass ratio of the powders to balls was 1:40. The vials were evacuated and filled with pure Ar at 4 Bar. As mentioned above, the ratio of the rotating speed, $K=w / W$, and the durations of the 
mechanical treatment were changed during the experiments (see Table 1). Since the mill possesses high rotation speed, water jet cooling of the vials was applied during the HEBM of the reactive mixtures.

The self-ignition temperature $\left(\mathrm{T}_{\mathrm{ig}}\right)$ for each as-milled powder was also measured. This method is described in details elsewhere [16]. Briefly, the disk-shaped samples, $3 \mathrm{~mm}$ in diameter and $0.5 \mathrm{~mm}$ height, were pressed. These samples were placed onto the $\mathrm{BN}$ crucible and heated at a rate of $50 \mathrm{~K} / \mathrm{s}$. The temperature-time history of the sample was measured by a $100 \mu \mathrm{m} \mathrm{W}$-Re thermocouple. At some point a sharp temperature increase occurs, which indicates that initiation of the exothermic reaction has occurred. The temperature value, which corresponds to the inclination point, is recognized as $\mathrm{T}_{\mathrm{ig}}$. At every stage of the experiment, the powder micro- and nano- structure, as well as phase composition were studied using FESEM (Magellan 400, FEI, USA) and XRD analysis (DRON-3, "Burevestnik", Russia). Crystallite size have been evaluated by Rietveld method [24, 25] using original software [26], instrumental broadening have been measured by means of stress-free Ge powder sample. The procedure was described in more details in the work [26].

\section{Results and discussion}

\subsection{Trajectories of Balls Motion}

Typical instantaneous images of the ball distributions inside the grinding vials, recorded by the high-speed video camera at different values of K, are shown at Figure 2. The statistical treatment of such frames allows us to conclude that, within the range of $\mathrm{K}$ values $0-1.9$, the balls can be sub-divided into two main groups: moving and stagnant. The moving balls continuously change their positions relative to the $\mathrm{X}-\mathrm{Y}$ coordinate system (Figure 1b), while the stagnant balls make only small oscillations in the vicinity of some steady position. Figure 3 shows experimentally measured trajectories of some moving balls, while the positions of the stagnant balls are indicated by the lightgray spots. It is worth noting that it was statistically proven that the number of stagnant balls decreases with increasing $\mathrm{K}$ and vanishes at $\mathrm{K}=2.0$, when all balls become be involved into motion. 
In turn, three types of ball movement were observed. At $\mathrm{K}<1.7$ the rolling of the balls primarily takes place and some "free fly" trajectories are also detected (Figure 3a,b). The free fly trajectory begins when the ball detaches from the compact group of the balls, and ends when it collides with other ball(s) or with the wall. It is worth noting that by increasing the $\mathrm{K}$ value from 0 to 1.7 , the number of "free fly" trajectories increases. A sharp change of the moving regime occurs at $K=1.8 \div 1.9$, when the free fly type of ball motion becomes dominant (Figure 3c,d). At $\mathrm{K} \geq 2.0$, the free fly motion changes into circular motion of the balls together with the inner walls of the grinding vial. For example, at $\mathrm{K}=2.0$ (Figure 3d) most of the balls form a rotating layer on the wall of the vial; if any balls fly out of this layer, they makes several "free flies" and return to the layer. Thus, in general, at each value of $\mathrm{K}$, a combination of two or three types of ball trajectories (stagnant, rolling, fly and circular) occurred, with one of them being most prevalent, i.e. rolling + stagnant at $\mathrm{K}=0-1.7$; flying + stagnant at $\mathrm{K}=1.8-1.9$; circular at $\mathrm{K} \geq 2.0$.

Distributions of the instantaneous velocities of the moving balls were measured with a frequency of $2000 \mathrm{~Hz}$ and the results are presented in Figure 4. Moderate values of the velocity, up to $\sim 4 \mathrm{~m} / \mathrm{s}$, with an average of $\sim 1-2 \mathrm{~m} / \mathrm{s}$, were observed for conditions with $\mathrm{K}<1.9$ (Fig. 4 a-c). The scattering of the measured values sharply increases when the $\mathrm{K}$ parameter reaches 1.9 (Fig.4 d,e). Some results of the statistical treatment of instantaneous accelerations of the balls are presented in Table 2. These data show that the acceleration values undergo significant variation. Indeed, an average instantaneous acceleration calculated in a time period of $0.5 \mathrm{~ms}$ (one video frame) for all $\mathrm{K}$ values is $330 \mathrm{G}$, (where $\mathrm{G}=9.8 \mathrm{~m} / \mathrm{s}^{2}$ - free fall acceleration on Earth), while the maximum acceleration can be as high as $2500 \mathrm{G}$.

\subsection{Structure and reactive properties of the as-milled mixtures}

\subsubsection{Ni-Al System}

Figure 5 shows the morphology of the initial and as-milled particles in $\mathrm{Ni}-\mathrm{Al}$ system. It can be seen that the initial $\mathrm{Ni}+\mathrm{Al}$ mixture consists of rounded $\mathrm{Al}$ particles and irregularly shaped agglomerates of small $\mathrm{Ni}$ particles (Fig.5 a,b). Note that only 3 min of HEBM was used for all 
experiments with this system. After milling, composite Ni/Al particles were formed (Fig.5 c,d). By increasing K up to 1.8 , the size of the cold-welded composite particles considerably decreases (Fig.5eh). The inner structure of these composite particles was described elsewhere [16], it was shown that they consisted of sub-micron layers of both $\mathrm{Ni}$ and $\mathrm{Al}$. At $\mathrm{K}=2.0$ the composite particles suddenly disappear, and the morphology of the mixture (Fig.5 j,k) becomes similar to that for the initial one.

$\mathrm{XRD}$ analysis revealed a significant broadening of the $\mathrm{Ni}$ and $\mathrm{Al}$ diffraction peaks for the powders fabricated in the $\mathrm{K}$ range 0 - 1.5, while a further increase of $\mathrm{K}$ leads to a gradual return of these parameters to the initial values (Fig. 6 and Table 3). The crystallite size decreases 10 times for the powders milled at $\mathrm{K}=1.5$, as compared to that for the initial mixture. However for as-milled powder at $\mathrm{K}=2.0$ these parameters coincide with the initial values (Table 3). No trace of any reaction products was found in any of the planetary ball milled Ni-Al mixtures.

The experimentally obtained values of self-ignition temperature $\left(\mathrm{T}_{\mathrm{ig}}\right)$ are shown in Table 3. It can be seen that the initial mixture has a $\mathrm{T}_{\text {ig }}$ around the eutectics in Ni-Al system (m.p. $639{ }^{\circ} \mathrm{C}$ ). Short term (3 min) HEBM at $\mathrm{K}$ in the range 0-1.5 leads to a gradual decrease of the self-ignition temperature, while $\mathrm{T}_{\mathrm{ig}}$ starts to increase with a further increase of $\mathrm{K}$ value.

\subsubsection{Ti-Si System}

Typical microstructures of the Ti+0.6Si mixtures before and after HEBM are shown in Figure 7. It can be seen that the initial mixture involves the round-like Ti particles and plate-like fragments of the Si particles. During HEBM with $\mathrm{K}$ in the range 0-1.9, the brittle Si particles are crushed into smaller fragments and cold-welded with ductile Ti to form Ti/Si composite particles. Again after milling at $\mathrm{K}=2.0$, the microstructure of the as-milled mixture is close to the initial one.

The XRD studies show (Table 3) that, similar to the Ni-Al system, the crystallite size of both precursors gradually decreases with an increasing $\mathrm{K}$ value from 0 to 1.5 and then increases, but being still much less than that for the initial mixture for powders milled at $\mathrm{K}=1.9$. Almost no change in the size of coherent scattering regions can be detected for as-milled powder at $\mathrm{K}=2.0$. However, some 
differences compared to the $\mathrm{Ni}-\mathrm{Al}$ system are also observed. It can be seen that 3 min milling at $\mathrm{K}=1.5$ leads to the formation of a large amount $(80 \%)$ of the product phase $\left(\mathrm{Ti}_{5} \mathrm{Si}_{3}\right)$.

For $\mathrm{K}=1.0, \mathrm{~T}_{\mathrm{ig}}$ of the as-milled products decreases to $680{ }^{\circ} \mathrm{C}$, which is almost twice less than that of the initial mixture $\left(1320^{\circ} \mathrm{C}\right)$. As mentioned above, a large amount of the product, $\mathrm{Ti}_{5} \mathrm{Si}_{3}$, was detected in the powder after milling at $\mathrm{K}=1.5$ and this mixture is not ignitable. A further increase of $\mathrm{K}$ leads to relatively high values of $\mathrm{T}_{\mathrm{ig}}\left(>1000^{\circ} \mathrm{C}\right)$. At $\mathrm{K}=2.0$, the structure and properties of the as-milled mixture are close to the initial one.

\subsubsection{Si-C System}

A longer milling time (15 minutes) was used for the $\mathrm{Si}+\mathrm{C}$ system. The microstructural transformation of this "brittle-brittle" type of mixture is shown in Figure 9. In the wide range of $\mathrm{K}=0 \div 1.8$, carbon transforms into an amorphous phase. Notable amounts of $\beta$-SiC were detected after activation at $\mathrm{K}=1.0 \div 1.8$ (Table 3 ).

The results for $\mathrm{T}_{\mathrm{ig}}$ are presented in Table 3. The mixture treated at $\mathrm{K}=0$ possesses an $\mathrm{T}_{\mathrm{ig}}$ close to the melting point of $\mathrm{Si}\left(\mathrm{T}_{\mathrm{m}}=1415^{\circ} \mathrm{C}\right)$, which is $100{ }^{\circ} \mathrm{C}$ less than that for the initial mixture $(1530$ ${ }^{\circ} \mathrm{C}$ ). The minimum ignition temperature was measured for the particles activated at $\mathrm{K}=1.5$, despite the fact that this mixture contains about $33 \%$ of the reaction product $(\beta-\mathrm{SiC})$ that was formed during HEBM. For particles fabricated during $\mathrm{HEBM}$ at $\mathrm{K}=1.9, \mathrm{~T}_{\mathrm{ig}}=1360{ }^{\circ} \mathrm{C}$, which is still below the $\mathrm{Si}$ melting point. Mixtures treated at $\mathrm{K}=2.0$ show similar characteristics to that of the initial sample.

The obtained experimental results reveal clear correlations between the type of ball trajectory and the milling parameter $(\mathrm{K})$, between the milling regime and microstructural transformations, as well as on the reactivity of the powder mixtures. It can be seen that, in general, these correlations are suitable for different kinds of binary powder mixtures, including brittle or ductile powders, or their combination. Below, the correlations are discussed in more detail.

In analyzing the obtained experimental results, let us first consider the concept of "milling efficiency" for reactive systems. In general, different parameters can be used for the efficiency evaluation. For example, the ignition temperature, i.e. a lower $\mathrm{T}_{\mathrm{ig}}$ indicates that the system was more 
effectively "mechanically activated". The amount of product produced in the jar during the milling can be used to measure efficiency; the more product formed during the treatment indicates that the reactive powder mixture more effectively interacted with the milling media (balls, walls). Finally, the changes in the crystalline size of the precursors, as compared to the initial parameters, can be a measure of the HEBM effectiveness. Analysis of data presented in Table 3, allows us to conclude that the three suggested, approaches for effectiveness evaluation strongly correlate with each other. Indeed, mechanically treated powders with the minimum ignition temperature have the smallest crystalline sizes. Also a decrease of crystalline size and of ignition temperature correlates with an increase of amount of product (if any) that is formed during milling. If the amount of product formed is too high then the system becomes incombustible, which does not contradict the conclusion about the correlations.

Based on the above context, it can be concluded that the most effective condition for the mechanical treatment of the investigated reaction systems is HEBM at $\mathrm{K}=1.5$, which corresponds predominantly to a rolling trajectory of the balls. In order to find relationships between effectiveness of the ball milling and ball trajectory, it is useful, based on the obtained data on the velocity and acceleration of the balls, to make estimations of the collision energy at different regimes.

The described ball motions correspond to the commonly recognized milling regimes: rolling of the balls corresponds to the cascading regime, free fly corresponds to the, so-called falling or cataracting regime, and circular motion relies to the centrifugal regime [27]. It is important to note that, a group of stagnant balls, which keep their positions in the rotating coordinate system (X,Y at Fig.1) for relatively long times, are also observed in the cascading $(\mathrm{K}=0-1.6)$ and falling $(\mathrm{K}=1.7-1.9)$ regimes.

The free-flying balls have constant velocity and zero acceleration in the laboratory coordinates, but they have high accelerations up to $549 \mathrm{~m} / \mathrm{s}^{2}\left(-a_{s}\right)$ in the rotating XY coordinate system. The stagnant balls remain in steady positions only in the mentioned above XY coordinate system. Relative to the laboratory system of coordinates, the balls move circularly with the rotational speed W (e.g. 694 
rpm) and their tangential velocity is $V_{s}=W R_{s}$, where $R_{s}$ is the distance between the stagnant ball and center of the sun wheel, which may vary from R-2r to R $(0.104 \mathrm{~m}$, see Table 1$)$. Thus in the laboratory coordinate, a stagnant ball located at the bottom position $(\mathrm{R})$ inside the grinding vial possesses velocity $\mathrm{V}_{\mathrm{s}}=2 \pi \mathrm{RW}=7.6 \mathrm{~m} / \mathrm{s}$ and corresponding centrifugal acceleration $\mathrm{a}_{\mathrm{s}}=(2 \pi \mathrm{W})^{2} \mathrm{R}=549 \mathrm{~m} / \mathrm{s}^{2}=56 \mathrm{G}$ system (see Fig,11). Finally, a stagnant ball that has contact with the wall (Fig.11), can slide or roll along the wall with a velocity of up to $8.7 \mathrm{~m} / \mathrm{s}$ (relative the wall) and, in the latter case, the ball rotates with speed $\omega=\mathrm{w}\left(\mathrm{r} / \mathrm{r}_{\mathrm{b}}\right)$. For the considered parameters of the planetary mill (Table 1 ), the rotating speed can be as high as $\omega=4601 / \mathrm{s}$.

Similar estimations for a ball "stuck" at the inner wall of the vial and making circular motion together with the vial (centrifugal regime; $\mathrm{K}=2$ ) in the $\mathrm{XY}$ system of coordinates gives tangential velocity $8.7 \mathrm{~m} / \mathrm{s}$ and radial acceleration $1900 \mathrm{~m} / \mathrm{s}^{2}=194 \mathrm{G}$. The instantaneous velocity and acceleration of such balls in the laboratory coordinate system can be calculated as the superposition of the corresponding vectors in the XY coordinate system and the circular motion of this system itself.

The obtained values of the ball velocities and accelerations allow us to estimate the level of interaction between the balls moving along different trajectories and the powder. Since the velocity of translational motion of the ball is in the range $1-10 \mathrm{~m} / \mathrm{s}$, the kinetic energy of the ball $\left(\mathrm{m}_{\mathrm{b}}=0.8 \mathrm{~g}\right.$ see Table 1) is in the range $0.0004 \div 0.04 \mathrm{~J}$. The energy of collision of the balls is usually considered as 1 $-2 \mathrm{~J}$ for high-energy ball mills and $0.1-0.2 \mathrm{~J}$ for low-energy ball mills [28]. Therefore, the collision energy of the moving balls, in our conditions, is small. The fraction of moving balls at $\mathrm{K}=0-1.5$ is also small compared to the number of balls inside the grinding vial because most balls are stagnate. The average acceleration of the moving balls is about $2200-3350 \mathrm{~m} / \mathrm{s}^{2}$ (Table 2). Thus, we may estimate that the average force affecting acceleration of the moving balls is $\mathrm{F}=\mathrm{m}_{\mathrm{b}} \mathrm{a}=1.8-2.7 \mathrm{~N}$. The largest observed accelerations $\sim 25000 \mathrm{~m} / \mathrm{s}^{2}$ give $\mathrm{F} \sim 20 \mathrm{~N}$. Note that in the above estimations it is assumed that the forces act continuously during $0.5 \mathrm{~ms}$, which is the exposure time of the high speed video recording. Although these values are only rough approximations, they show that the moving balls apparently do not provide large enough forces to destroy the crystal structure of precursors (e.g. 
$\mathrm{Ni}$ or Ti). Also, the total time of such impacts is only a very small part of the overall duration of the process.

The stagnant balls form a main group of grinding bodies, where all balls are in continuous contact with each other. The weight of each ball rotating at $\mathrm{W}=6941 / \mathrm{min}$ is $\sim 0.4 \mathrm{~N}$, due to centrifugal acceleration. However, cooperative simultaneous act of hundreds of balls stacked in this group creates strong continues pressure between the neighboring balls and/or between the balls and the wall of the vial. Friction between the ball surfaces is probably the main mechanism of interaction between this group of the grinding bodies and the powder. As it was previously shown [16], that ductile metal (Al, Ni, Ti) particles stacked on the surface of the balls and on the wall, undergo severe shear deformation due to the dry friction. In turn, the brittle particles ( $\mathrm{Si}, \mathrm{C}$ ) are grinded in this zone due to the friction and small relative motion of the stagnant balls.

Based on the above it can be concluded that the stagnant balls play a leading role in the mechanical activation of the reactive powder mixtures. The "activation" occurs in the thin surface layer of the composite particles formed by cold welding of the reactants, due to shear deformation by dry friction. The largest number of stagnant balls corresponds to the cascading regime $(\mathrm{K}=0-1.5)$, which explains the effectiveness of powder activation at this regime with highest speed of the jar $(\mathrm{K}=1.5)$. Falling balls $(\mathrm{K}=1.8-1.9)$ mostly affect the size of the composite agglomerates, because the impact of these balls limit the growth of the composite layers on the surface of the balls and/or on the wall of the jar. Centrifugal balls $(\mathrm{K}=2)$ make negligible activating effect due to low collision energy.

\section{Conclusion}

The direct relationships between the kinematics of ball motion during high energy planetary ball milling, structure and morphology of the milled powder reactive mixtures $(\mathrm{Ni}+\mathrm{Al}, \mathrm{Si}+\mathrm{C}$, $\mathrm{Ti}+0.6 \mathrm{Si}$ ), and the reactive properties of these mixture were found by experimental research. The most efficient HEBM regime was achieved when the ratio between the rotating speed of the milling vials, w, and the sun disk, $\mathrm{W}$, was $\mathrm{K}=\mathrm{w} / \mathrm{W}=1.5$, which corresponds to predominantly rolling trajectories of the moving balls and a large fraction of stagnant balls inside the vial. At these regimes, the fastest 
transformation of crystal structure and morphology of the particles was observed, along with the most significant changes in the reactive properties, e.g., a decrease of the self-ignition temperature. A hypothesis has been suggested that the stagnant balls may play a leading role in the mechanical activation of the reactive powder mixtures.

\section{Acknowledgements}

This work was carried out with financial support from the Ministry of Education and Science of the Russian Federation in the framework of Increase Competitiveness Program of NUST «MISiS» (no. K2-2014-001). It was also partially supported by the Department of Energy, National Nuclear Security Administration, under Award Number DE-NA0002377 and funding from the Defense Threat Reduction Agency (DTRA), Grant Number HDTRA1-10-1-0119. Counter-WMD basic research program, Dr. Suhithi M. Peiris, program director is gratefully acknowledged.

\section{References}

[1] C.C. Koch, J.D. Whittenberger, Mechanical milling/alloying of intermetallics, Intermetallics 4 (1996) 339-355.

[2] C. Suryanarayana, Mechanical Alloying and Milling, Marcel Dekker, New York 2004.

[3] C.C. Koch, Synthesis of nanostructured materials by mechanical milling: problems and opportunities, Nanostructured materials 9 (1997) 13-22.

[4] L. Takacs, Self-sustaining reactions induced by ball milling, Progress in Materials Science 47 (2002) 355-414.

[5] F. Maglia, U. Anselmi-Tamburini, C. Deidda, F. Delogu, G. Cocco, Z.A. Munir, Role of mechanical activation in SHS synthesis of TiC, J. Mater. Sci., 39 (2004) 5227-5230.

[6] E.L. Dreizin, Metal-based reactive nanomaterial, Progress in Energy and Combustion Science 35 (2009) 141-167.

[7] N.F. Shkodich, A.S. Rogachev, S.G. Vadchenko, N.V. Sachkova, R. Chassagnon, Reactivity of mechanoactivated Ni-Al blends, Int. J. SHS 21(2) (2012) 104-109.

[8] M.A. Korchagin, T.F. Grigorieva, A.P. Barinova, N.Z. Lyakhov, The effect of mechanical treatment on the rate and limits of combustion in SHS processes, Int. J. SHS, 9(3) (2000) pp.307320.

[9] F. Bernard, E. Gaffet, Mechanical alloying in the SHS research, Int. J. SHS, 10(2) (2001) 109-132.

[10] T. Grigorieva, M. Korchagin, N. Lyakhov, Combination of SHS and mechanochemical synthesis for nanopowder technologies, KONA, 20 (2002) 144-158. 
[11] F. Maglia, U. Anselmi-Tamburini, G. Cocco, M. Monagheddu, N. Bertolino, Z.A. Munir, Combustion synthesis of mechanically activated powders in the Ti-Si system, J. Mater. Res. 16(4) (2001) 1074-1082.

[12] A.S. Rogachev, N.A. Kochetov, N.V. Sachkova, V.V. Kurbatkina, E.A. Levashov, P.S. Grinchuk, O.S. Rabinovich, F. Bernard, Microstructural aspects of gasless combustion of mechanically activated mixtures. I. High-speed microvideorecording of the Ni-Al composition, Combust., Explos., Shock Waves, 42 (2006) 421-429.

[13] A.G. Merzhanov, Self-propagating high-temperature synthesis: Twenty years of search and findings, in: Z. Munir, J.B. Holt (eds.), Combustion and Plasma Synthesis of High-Temperature Materials, VCH Publ., N.Y., 1990, pp. 1-53.

[14] A. Varma, A.S. Rogachev, A.S. Mukasyan, S. Hwang, Combustion synthesis of advanced materials: principles and applications, in: James Wei (editor-in chief), Advances in chemical engineering, Academic Press, 1998, v.24, pp.79-226.

[15] A.S. Mukasyan, Ya-Cheng Lin, A.S. Rogachev, D.O. Moskovskikh, Direct Combustion Synthesis of Silicon Carbide Nanopowder from the Elements, J. Amer. Ceram. Soc. 96 (2013) 111-117.

[16] A.S. Rogachev, N.F. Shkodich, S.G. Vadchenko, F. Baras, D.Yu. Kovalev, S. Rouvimov, A.A. Nepapushev, A.S. Mukasyan, Influence of the high energy ball milling on structure and reactivity of the $\mathrm{Ni}+\mathrm{Al}$ powder mixture, J. Alloys Compd. 577 (2013) 600-605.

[17] KV. Manukyan, Ya-C. Lin, S. Rouvimov, PJ. McGinn, A.S. Mukasyan, Microstructure-reactivity relationship of Ti-C reactive nanomaterials, J. Appl. Phys. 113 (2), 024302-024302-10 (2013).

[18] M. Abdellaoui, E. Gaffet, The physics of mechanical alloying in a Planetary ball mill: mathematical treatment, Acla Metall. Mater. 43 (1995) 1087-1098.

[19] Y.T. Feng, K. Han, D.R.J. Owen, Discrete element simulation of the dynamics of high energy planetary ball milling processes, Mater. Sci. Eng. A 375-377 (2004) 815-819.

[20] Hiroshi Mio, Junya Kano, Fumio Saito, Kantaro Kaneko, Effect of rotational direction and rotation-to-revolution speed ratio in planetary ball milling, Mater. Sci. Eng. A332 (2002) 75-80.

[21] A.G. Ermilov, E.B. Bogatyreva, Preliminary mechanoactivation, MISiS Publishig House, Moscow, 2012 (in Russian).

[22] P. LeBrun, L. Froyen, L.Delaey, The modeling of the mechanical alloying process in a planetary ball mill: comparison between theory and in-situ observations, Mater. Sci. Eng. A161 (1993) 75-82.

[23] S. Rosenkranz, S. Breitung-Faes, W. Kwade, Experimental investigation and modeling of the ball motion in planetary ball mills, Powder Technology 212 (2011) 224-230.

[24] H.M.Rietveld Line profiles of neutron powder-diffraction peaks for structure refinement, Acta Crystallogr., 22 (1967), 151-152.

[25] H.M.Rietveld The Rietveld method, Phys. Scr., 89 (2014), 098002(1-6). 
[26] E.V.Shelekhov, T.A.Sviridova. Programs for X-ray Analysis of Polycrystals. Metal Science and Heat Treatment, v.42 (2000), No.8, pp.309-313.

[27] S.Bernotat, K.Schonert, (1998) Size reduction. In: “ Ullmann's Encyclopedia of Industrial Chemistry. VCH Verlagsgesellschaft, Weinheim, Vol. B2, pp. 5.1-5.39.

[28] A.S. Rogachev, A.S. Mukasyan, Combustion of heterogeneous nanostructural systems (review), Combust., Explos., Shock Waves, 46(3) 2010 243-266. 


\section{Tables}

Table 1. Parameters of the HEBM used in this work.

\begin{tabular}{l|l} 
Radius of the sun wheel, $\mathrm{R}$ & $0.104 \mathrm{~m}$ \\
Radius of the grinding chamber, $\mathrm{r}$ & $0.040 \mathrm{~m}$ \\
$\begin{array}{l}\text { Distance between the axle of the sun wheel and the axle of the } \\
\text { grinding chamber, R-r }\end{array}$ & $0.064 \mathrm{M}$ \\
Parameter M=(R-r)/r & 1.6 \\
Radius of the milling ball, $\mathrm{r}_{\mathrm{b}}$ & $0.003 \mathrm{~m}$ \\
Mass of the milling ball (steel), $\mathrm{m}$ & $0.8 \mathrm{~g}$ \\
Rotating speed of the sun wheel, W & $694 \mathrm{~min}^{-1}$ \\
Rotating speed of the grinding chamber, w & $0-1388 \mathrm{~min}^{-1}$ \\
Parameter K=w/W & $0-2.0$ \\
Volume fraction of the balls inside the grinding chamber & 0.35 \\
Duration of milling & $3-15 \mathrm{~min}$ \\
\hline
\end{tabular}

Table 2. Statistical values of the balls acceleration.

\begin{tabular}{cccc}
\hline Parameter K & Mean, $\mathrm{m} / \mathrm{s}^{2}$ & Minimum, $\mathrm{m} / \mathrm{s}^{2}$ & Maximum, $\mathrm{m} / \mathrm{s}^{2}$ \\
\hline 1.0 & 3910 & 0 & 20880 \\
1.5 & 3440 & 0 & 24730 \\
1.8 & 2760 & 0 & 25280 \\
1.9 & 3350 & 0 & 19820 \\
2.0 & 2970 & 0 & 17720 \\
\hline
\end{tabular}

Table 3. Some properties of the powder mixtures before and after HEBM

\begin{tabular}{|c|c|c|c|c|c|c|c|c|c|c|c|c|}
\hline \multirow{3}{*}{$\begin{array}{c}\mathrm{K} \\
\text { value }\end{array}$} & \multicolumn{4}{|c|}{$\mathrm{Ni}+\mathrm{Al}$ mixture } & \multicolumn{4}{|c|}{$\mathrm{Ti}+0.6 \mathrm{Si}$ mixture } & \multicolumn{4}{|c|}{$\mathrm{Si}+\mathrm{C}$ mixture } \\
\hline & \multicolumn{2}{|c|}{$\begin{array}{l}\text { Crystallite size } \\
\text { by XRD, nm }\end{array}$} & \multirow{2}{*}{$\begin{array}{l}\text { wt.\% } \\
\text { pro- } \\
\text { duct } \\
\text { phase } \\
\text { XRD }\end{array}$} & \multirow{2}{*}{$\mathrm{T}_{\mathrm{ig}},{ }^{\circ} \mathrm{C}$} & \multicolumn{2}{|c|}{$\begin{array}{c}\text { Crystallite size } \\
\text { by XRD, nm }\end{array}$} & \multirow{2}{*}{$\begin{array}{l}\text { wt.\% } \\
\text { pro- } \\
\text { duct } \\
\text { phase, } \\
\text { XRD }\end{array}$} & \multirow{2}{*}{$\mathrm{T}_{\mathrm{ig}},{ }^{\circ} \mathrm{C}$} & \multicolumn{2}{|c|}{$\begin{array}{c}\text { Crystallite size } \\
\text { by XRD, nm }\end{array}$} & \multirow{2}{*}{$\begin{array}{l}\text { wt. } \% \\
\text { pro- } \\
\text { duct } \\
\text { phase, } \\
\text { XRD }\end{array}$} & \multirow{2}{*}{$\mathrm{T}_{\mathrm{ig}},{ }^{\circ} \mathrm{C}$} \\
\hline & $\mathrm{Ni}$ & $\mathrm{Al}$ & & & $\mathrm{Ti}$ & $\mathrm{Si}$ & & & $\mathrm{Si}$ & $\mathrm{C}$ & & \\
\hline initial & 190 & 300 & 0 & 640 & 130 & $>500$ & 0 & 1230 & 690 & 100 & 0 & 1560 \\
\hline 0 & 75 & 75 & 0 & 490 & 40 & 40 & 0 & 760 & 50 & N/A* & 0 & 1450 \\
\hline 1.0 & 40 & 40 & 0 & 360 & 20 & 25 & 0 & 680 & 15 & N/A* & 7 & 1380 \\
\hline 1.5 & 30 & 30 & 0 & 350 & 15 & 20 & 80 & $\begin{array}{c}\text { no } \\
\text { ignition }\end{array}$ & 10 & N/A* & 30 & 1130 \\
\hline 1.8 & 90 & 140 & 0 & 550 & 40 & 80 & 0 & 1050 & 15 & N/A* & 40 & 1360 \\
\hline 2.0 & 150 & 300 & 0 & 600 & 220 & $>500$ & 0 & 1070 & 250 & 40 & 0 & 1490 \\
\hline
\end{tabular}

*amorphous phase 


\section{Figure captions}

Figure 1. High-speed video frames of the rotating planetary mill. $K=1,0$ (a) and $K=1,9$ (b).

Figure 2. Instant balls distribution in the grinding vials depending on $K$ values: $K=1.0$ (a); 1.5 (b); 1.8 (c); 1.9 (d); 2.0 (e).

Figure 3. Typical trajectories of the moving balls: $\mathrm{K}=1.0$ (a); 1.5 (b); 1.8 (c); 1.9 (d); 2.0 (e).

Figure 4. Statistics on the velocities of the moving balls: $K=1.0$ (a); 1.5 (b); 1.8 (c); 1.9 (d); 2.0 (e).

Figure 5. SEM images of the $\mathrm{Ni}+\mathrm{Al}$ mixture before and after HEBM (3 min), depending on $\mathrm{K}$ parameter.

Figure 6. XRD spectra of the $\mathrm{Ni}+\mathrm{Al}$ mixtures before and after HEBM (3 min), depending on $\mathrm{K}$ parameter.

Figure 7. SEM images of the Ti+0.6Si mixture before and after HEBM (3 min), depending on K parameter.

Figure 8. XRD spectra of the Ti+0.6Si mixtures before and after HEBM (3 min), depending on $\mathrm{K}$ parameter. Symbol $\boldsymbol{\nabla}$ indicates XRD lines of the hexagonal phase $\mathrm{Ti}_{5} \mathrm{Si}_{3}$.

Figure 9. SEM images of the $\mathrm{Si}+\mathrm{C}$ mixture before and after HEBM (15 min), depending on $\mathrm{K}$ parameter.

Figure 10. XRD spectra of the $\mathrm{Si}+\mathrm{C}$ mixtures before and after HEBM (15 min), depending on $\mathrm{K}$ parameter.

Figure 11. Schematic draw of velocities and accelerations of the grinding balls. 
FIGURES

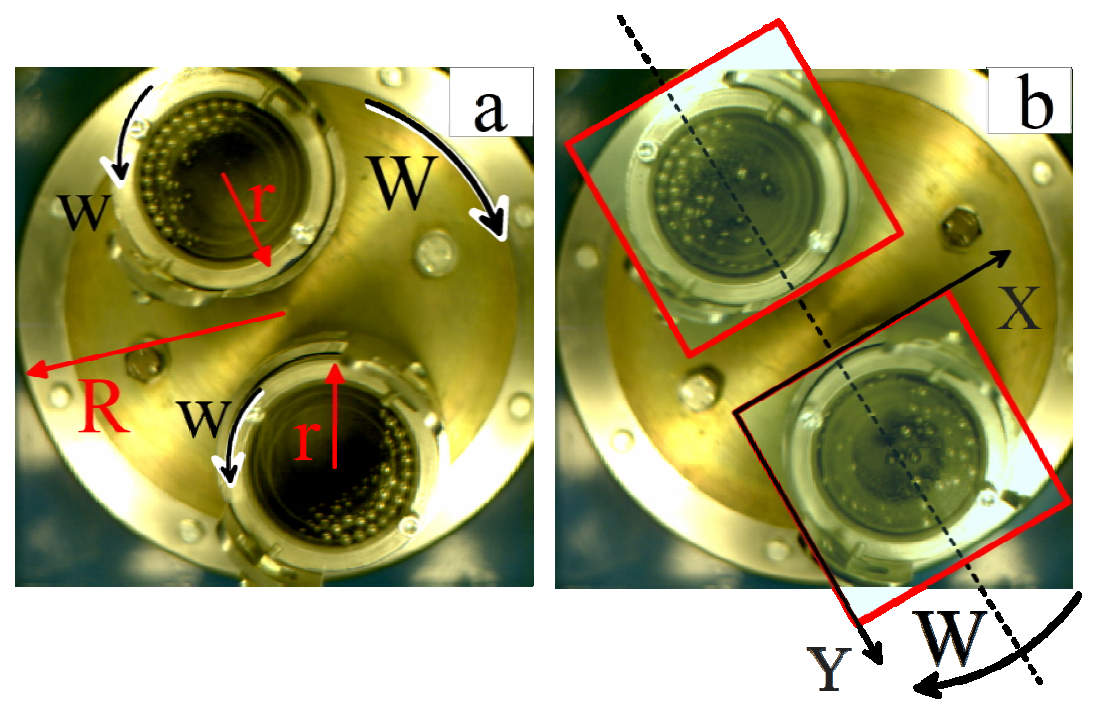

Figure 1.
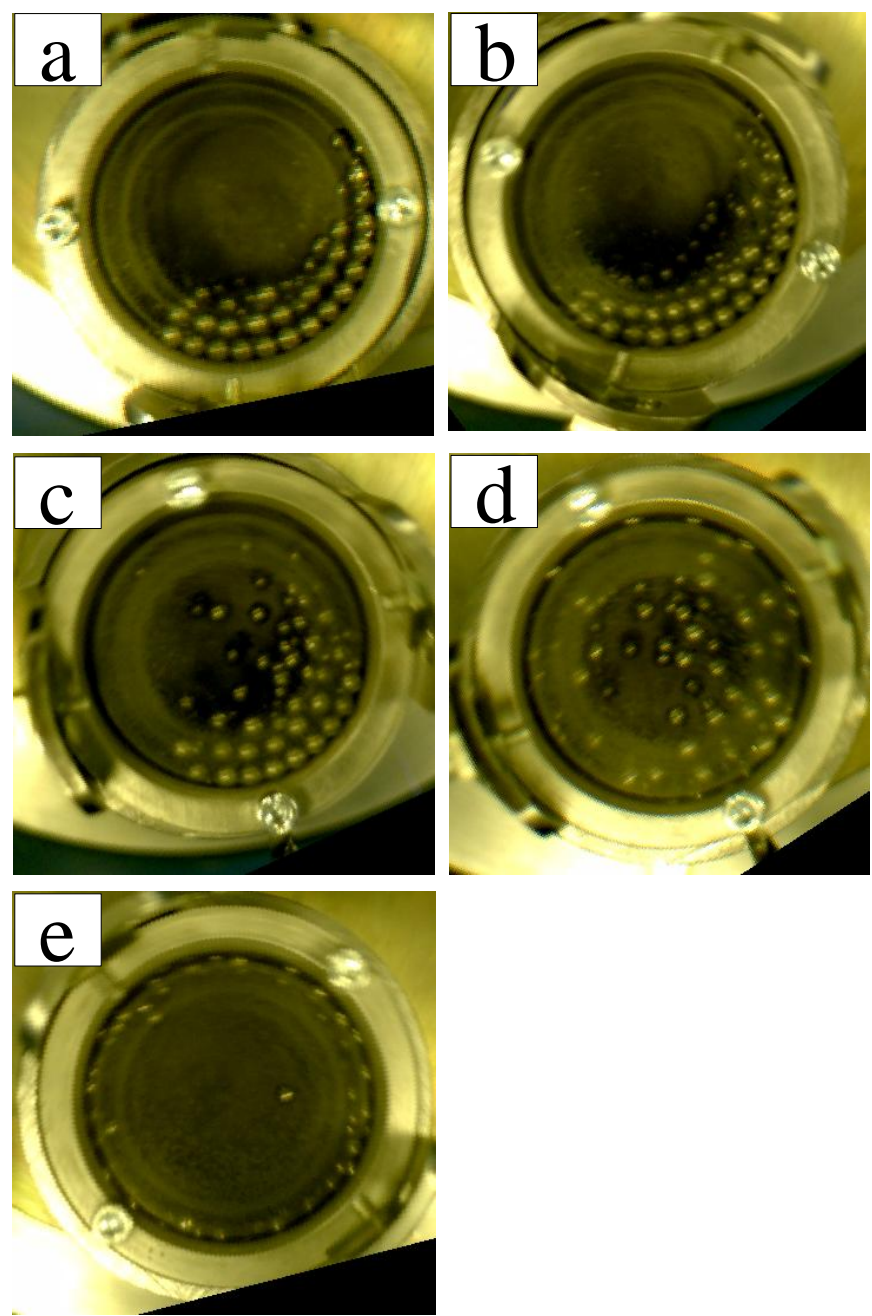

Figure 2 

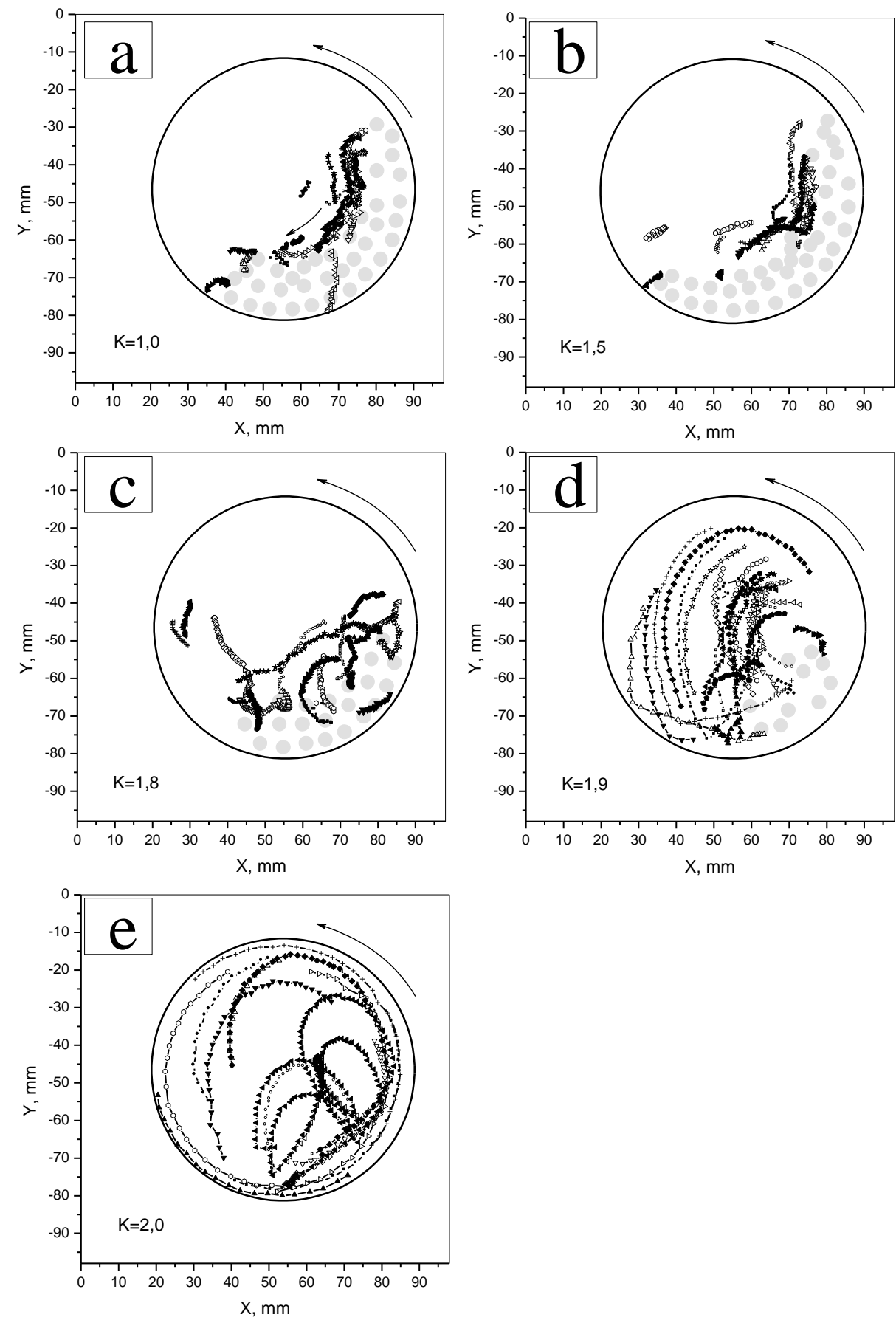

Figure 3 

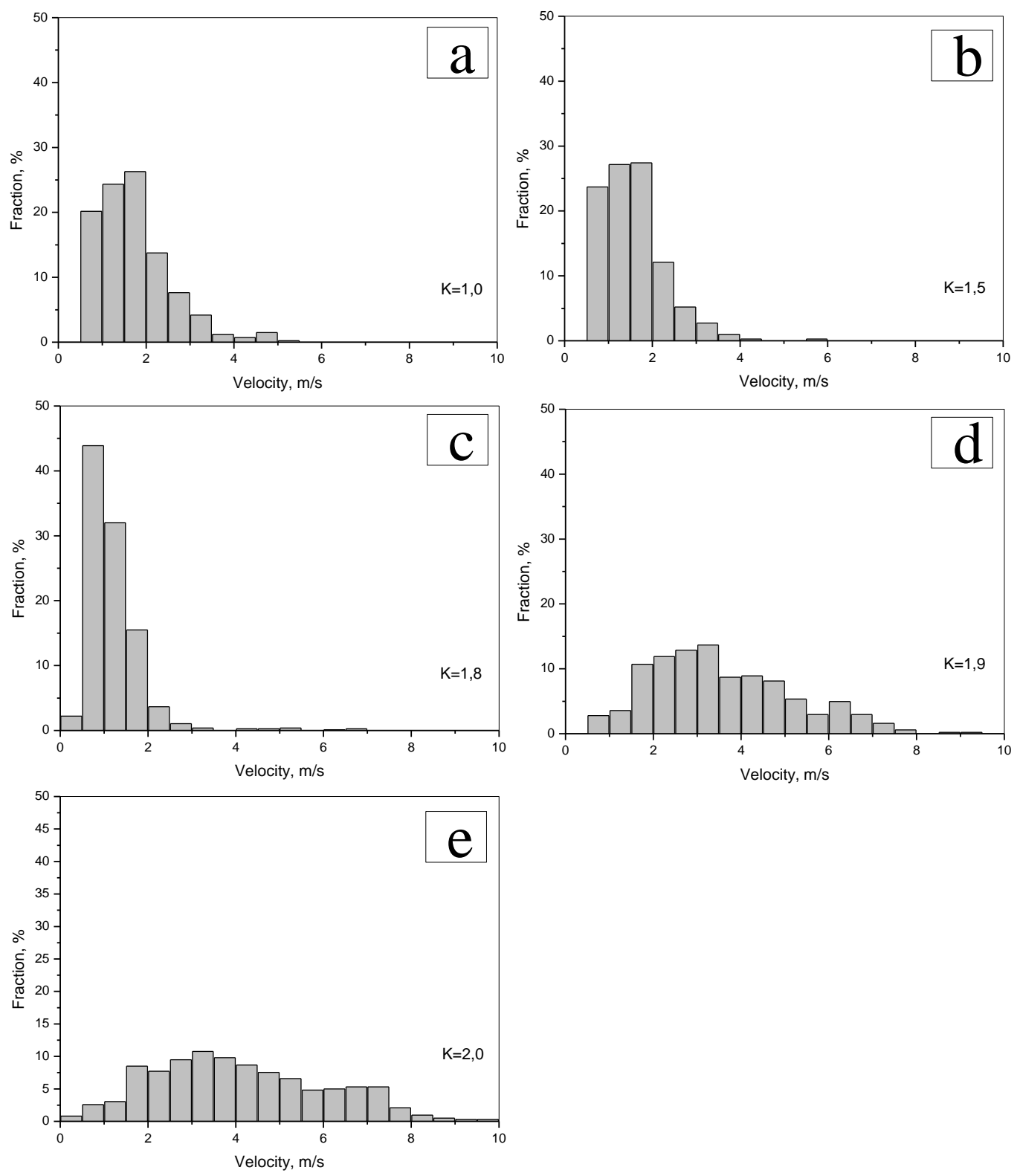

Figure 4. 

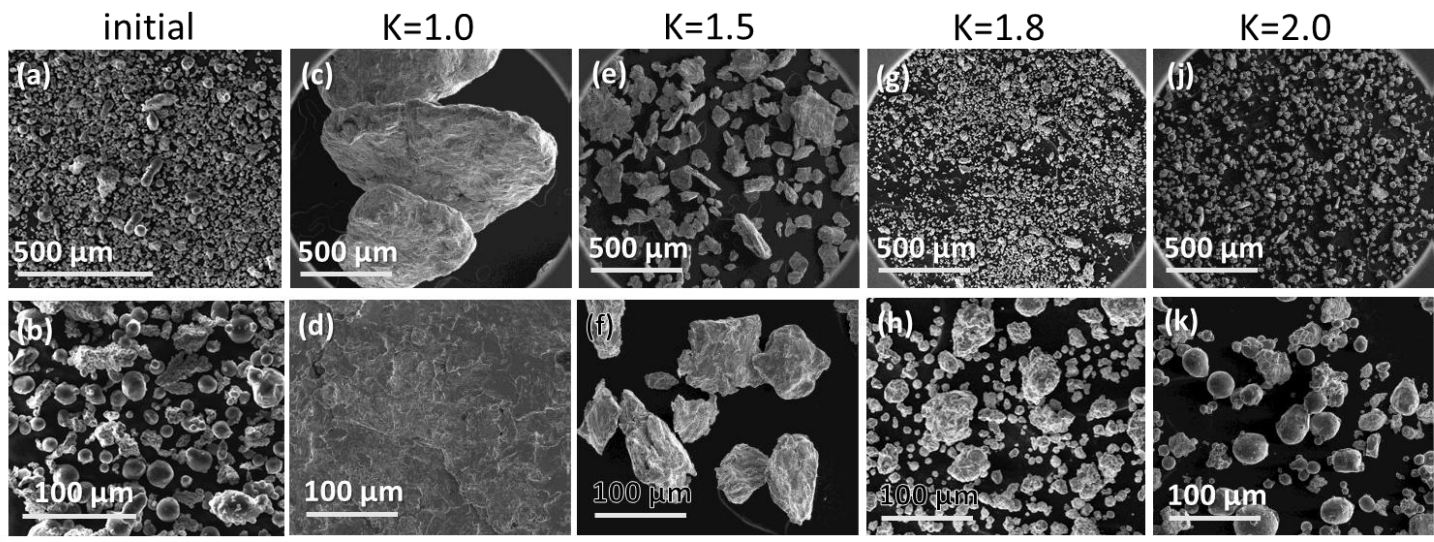

Figure 5.

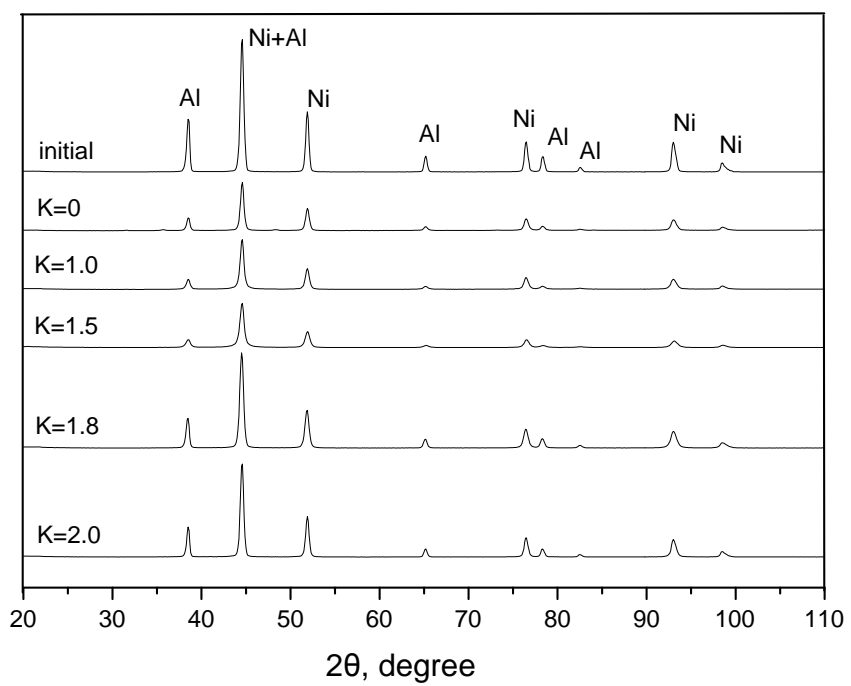

Figure 6. 

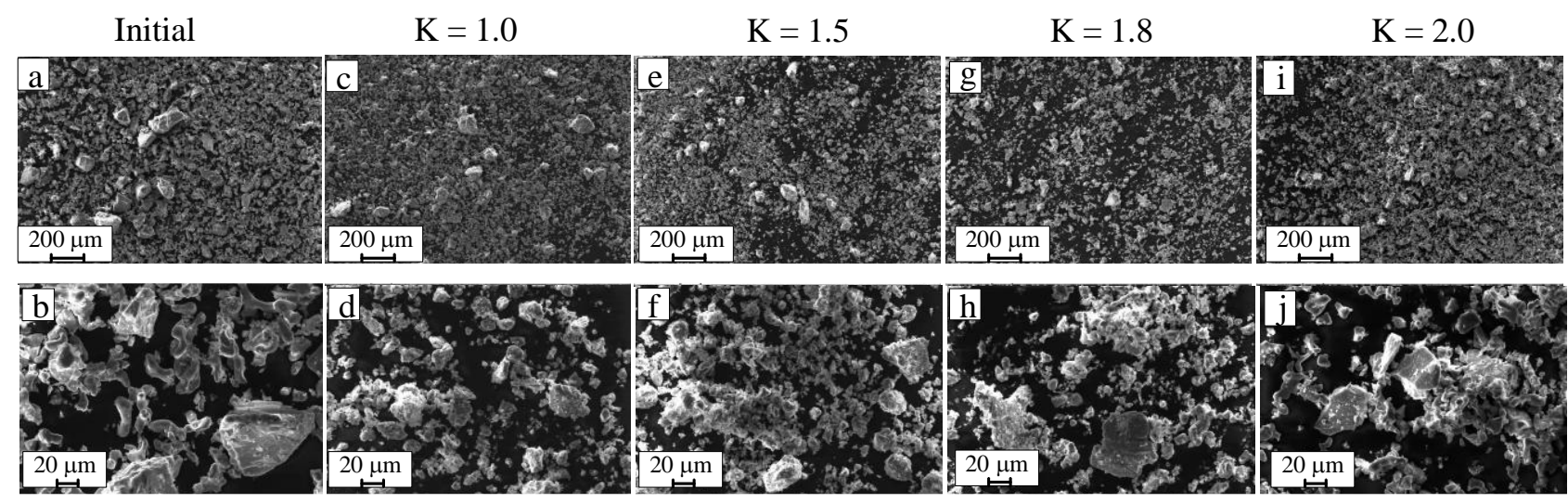

Figure 7.

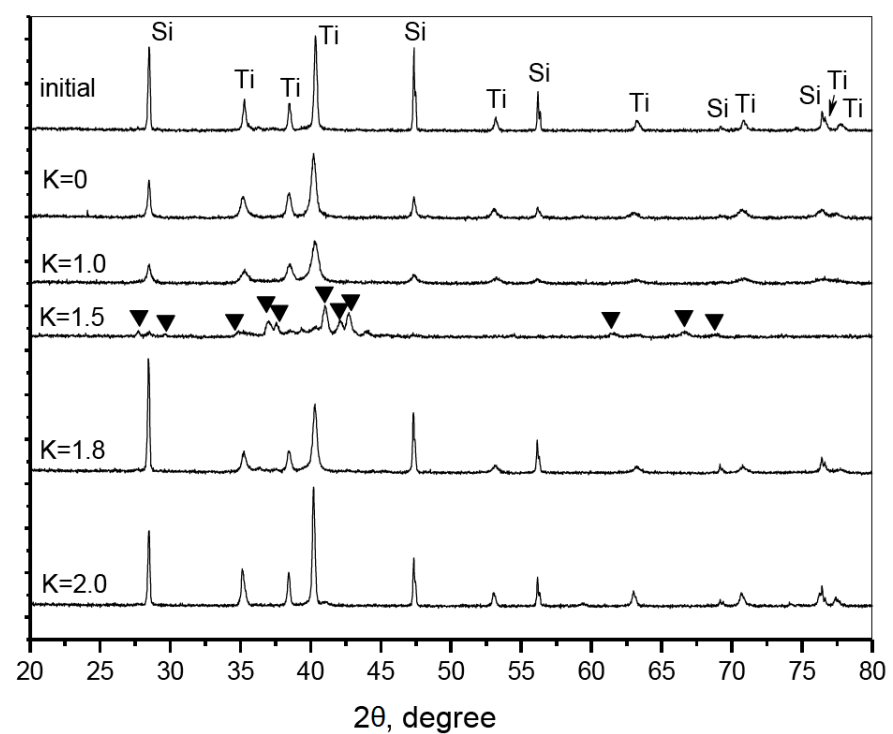

Figure 8. 

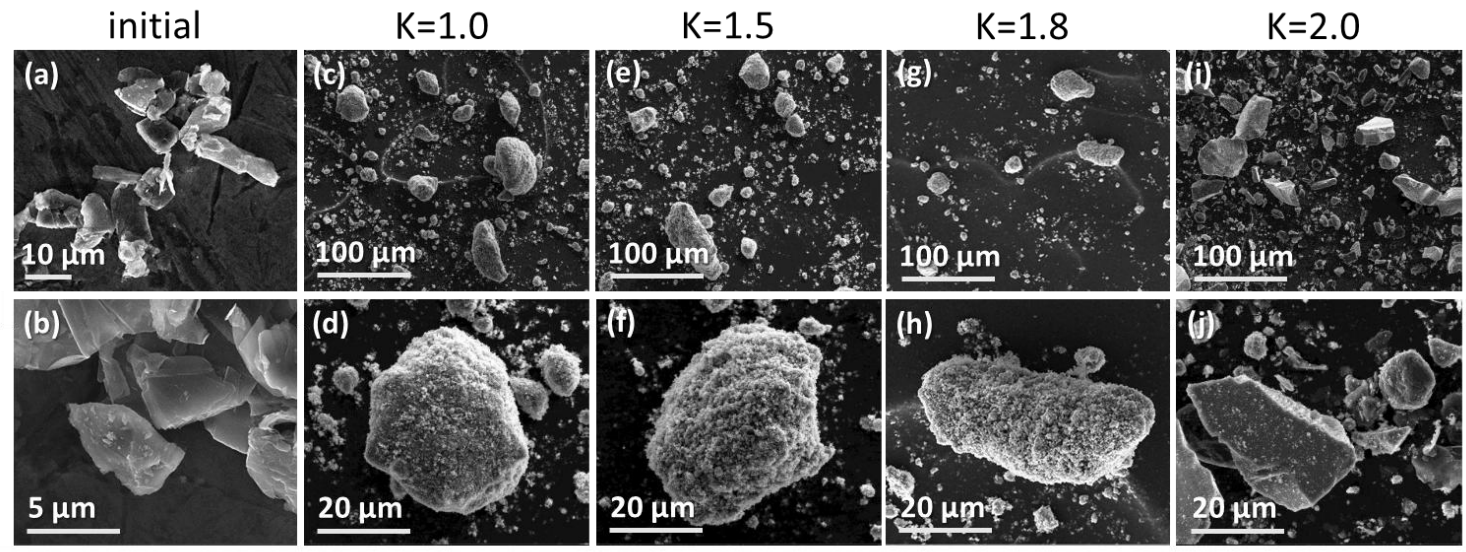

Figure 9

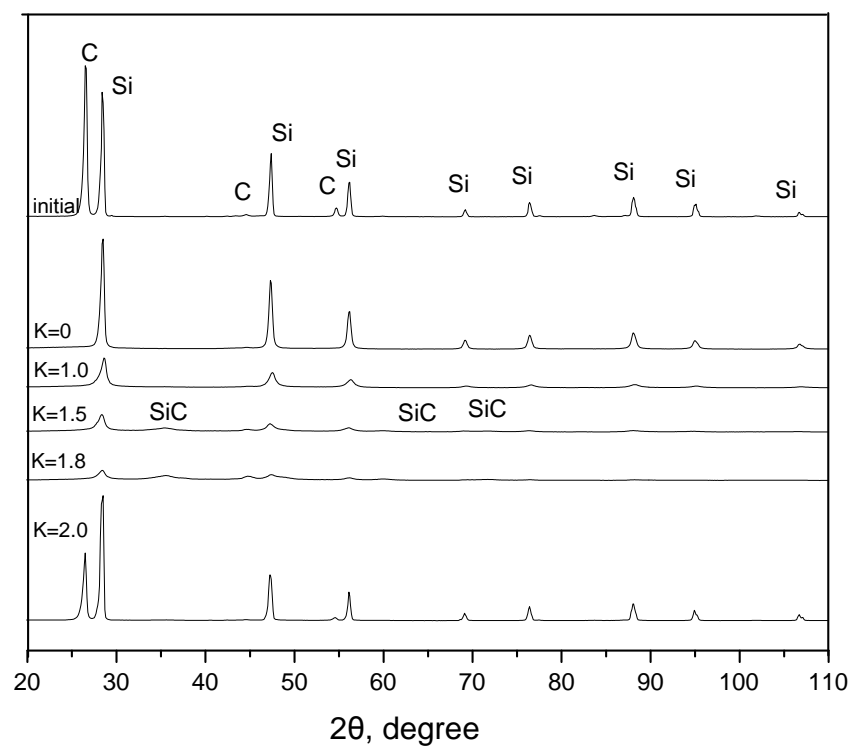

Figure 10. 


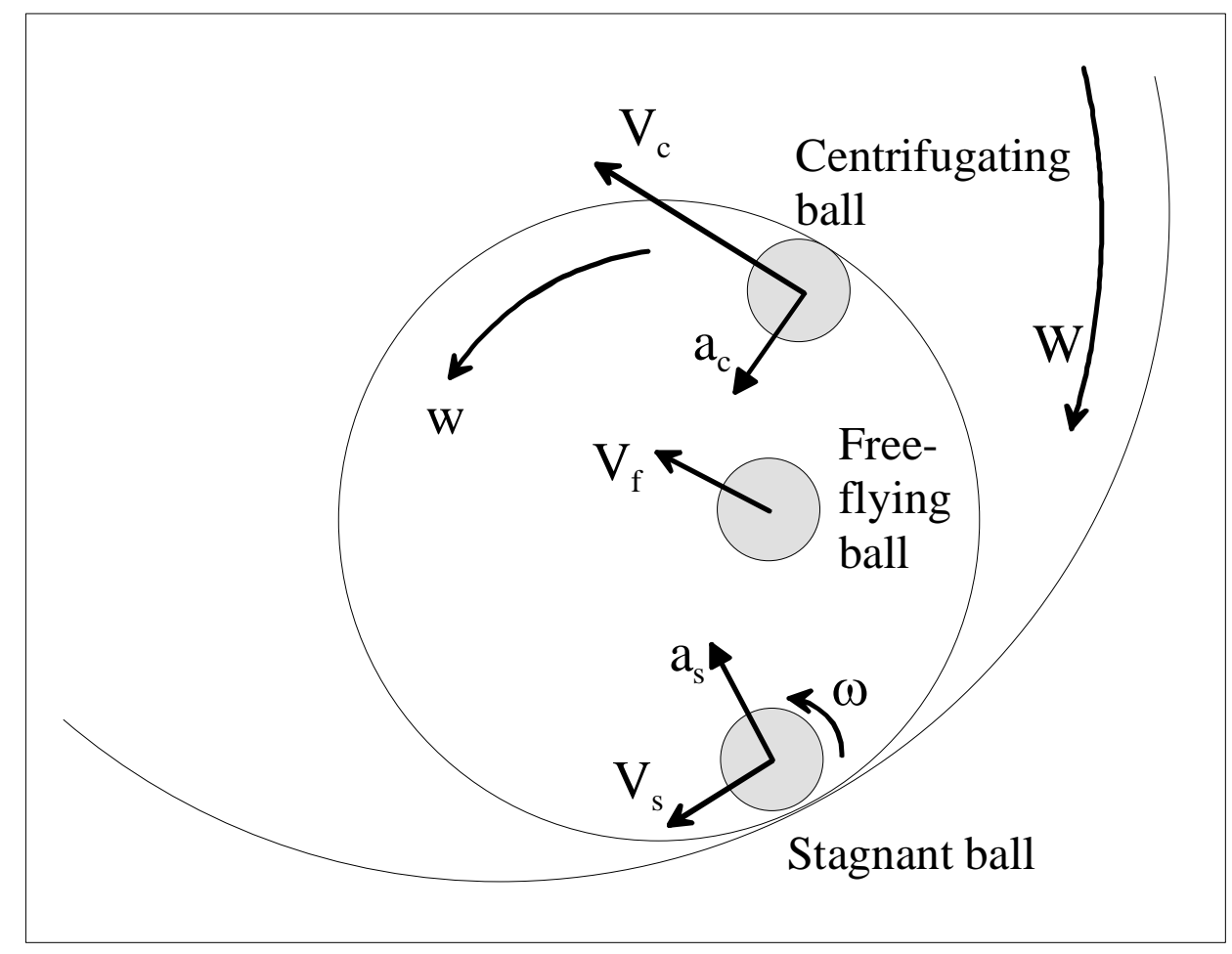

Figure 11. 


\section{Graphical Abstract}

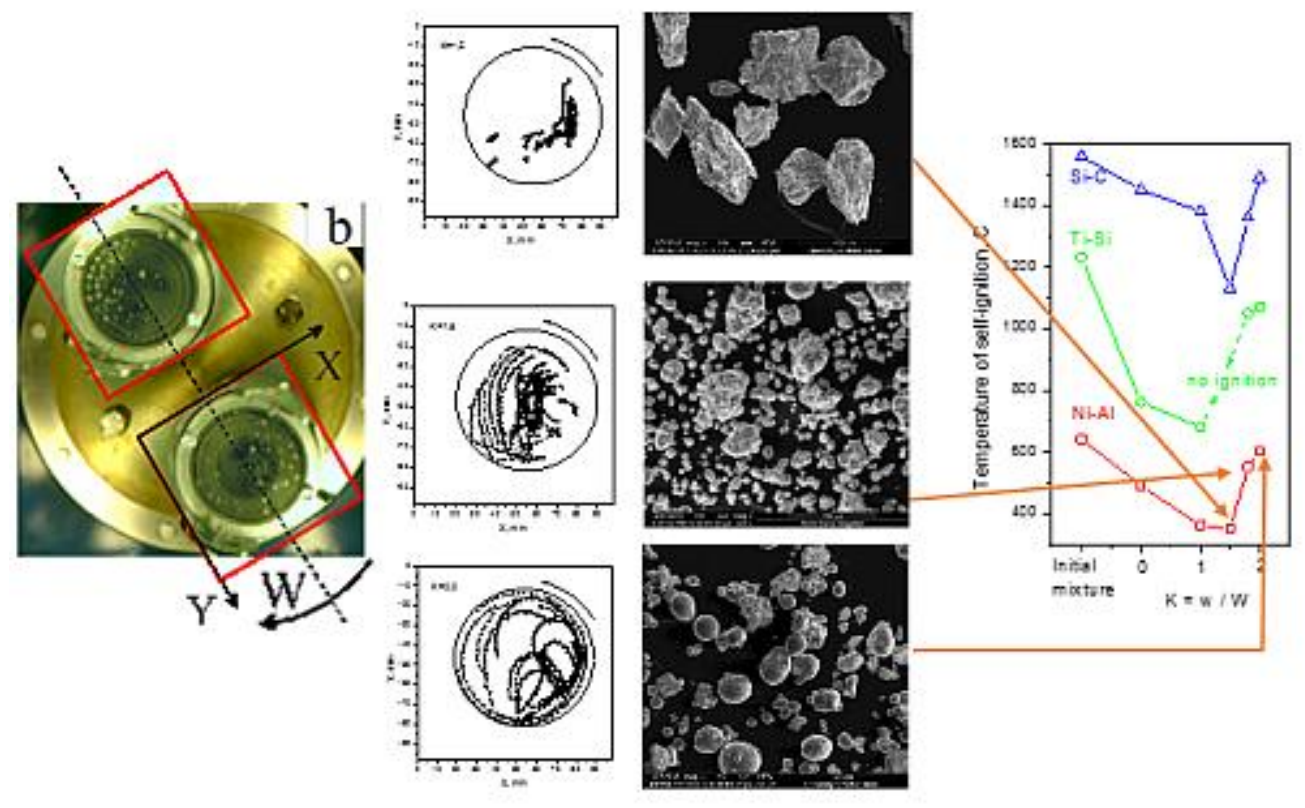

